demonstrated that the hydrophobic groove is conserved across BoNT/B and BoNT/G, but differs in other neurotoxins.

Gangliosides are crucial for the entry of BoNT/B into cells and support the binding of BoNT/B with synaptotagmins. To investigate BoNT/B recognition of gangliosides and Sytll, the authors docked a trisialoganglioside $\left(\mathrm{G}_{\mathrm{T} 1 \mathrm{~b}}\right)$ analog into the ganglioside binding site of sialyllactose in complex with BoNT/B. The results showed that $\mathrm{G}_{T 1 b}$ s binding site is more extensive than previously thought, and might form contacts with both synaptotagmins and BoNT/B.

These studies describe the structure of the BoNT/B-Sytll complex, and present a structural basis for the double-receptor hypothesis. These findings could, therefore, aid the development of pharmacological inhibitors that block the binding of toxins to cell-surface receptors.

Original articles Jin R et al. (2006) Botulinum neurotoxin $B$ recognizes its protein receptor with high affinity and specificity. Nature 444: 1092-1095

Chai Q et al. (2006) Structural basis of cell surface receptor recognition by botulinum neurotoxin B. Nature 444: 1096-1100

\section{Combination salvage chemotherapy for refractory germ cell tumors}

The majority of patients with disseminated testicular cancer can be cured with first-line cisplatin-based combination chemotherapy. Some patients do not respond to either firstline or standard salvage chemotherapy, however; new treatments are needed for these patients, whose prognosis is poor.

Bedano et al. reported the results of a Phase II study of cisplatin and epirubicin chemotherapy in patients with refractory germ cell tumors. The patients had worsening disease after initial cisplatin-based combination therapy, and their tumors were unresponsive to standard salvage chemotherapy.

The study included 30 male patients (median age 36 years). Of these, 21 had experienced late relapses more than 2 years after first-line chemotherapy. They were treated with $90 \mathrm{mg} / \mathrm{m}^{2}$ epirubicin administered intravenously on day 1 and $20 \mathrm{mg} / \mathrm{m}^{2}$ intravenous cisplatin on days $1-5$, repeated every 21 days, for a maximum of four cycles. Toxic effects were predominantly hematologic. A response to treatment was seen in 17 of 30 patients, and the median survival was 14.5 months. Seven patients had no evidence of disease at their final assessment (25-48 months after treatment). Two patients became disease-free after chemotherapy alone, and four became disease-free after chemotherapy followed by surgery.

The authors conclude that this combination of cisplatin and epirubicin offers the possibility of prolonged survival in patients with refractory germ cell tumors, with an acceptable toxicity profile. Combination chemotherapy followed by resection could offer patients who were not surgical candidates at relapse the chance to achieve disease-free status.

Original article Bedano PM et al. (2007) Phase II study of cisplatin plus epirubicin salvage chemotherapy in refractory germ cell tumors. J Clin Oncol 24: 5403-5407

\section{Subureteral copolymer injection is not cost-effective as initial therapy for VUR}

If medical treatment of vesicoureteral reflux (VUR) is unsuccessful, patients are offered ureteral reimplantation or subureteral injectionsfor example, of dextranomer-hyaluronic acid copolymer. Benoit and colleagues constructed models to assess whether these injections were a cost-effective first-line treatment for VUR, compared with standard treatment.

The cost-benefit model assumed that VUR was diagnosed by ultrasonography and voiding cystourethrography. Standard treatment comprised prophylactic antibiotics, and annual follow-up evaluations; patients with VUR who experienced breakthrough urinary-tract infections and those who still had VUR 5 years after diagnosis were offered ureteral reimplantation. Alternative treatment comprised subureteral injections of dextranomer-hyaluronic acid copolymer, given at diagnosis. Patients whose VUR did not resolve underwent either immediate ureteral reimplantation or standard treatment.

Overall, standard treatment was the most cost-effective first-line therapy for VUR, and was also the most cost-effective option when low-grade VUR (grades I-III), and bilateral and unilateral VUR were analyzed separately. By contrast, ureteral reimplantation was the most cost-effective first-line therapy for high-grade VUR (grades IV-V), although the authors noted that this finding depended on region-specific 